Peter Ullrich

\title{
Videoüberwachung von Demonstrationen und die Definitionsmacht der Polizei. Zwischen Objektivitätsfiktion und selektiver Sanktionierung
}

Journal article | Accepted manuscript (Postprint)

This version is available at https://doi.org/10.14279/depositonce-9981

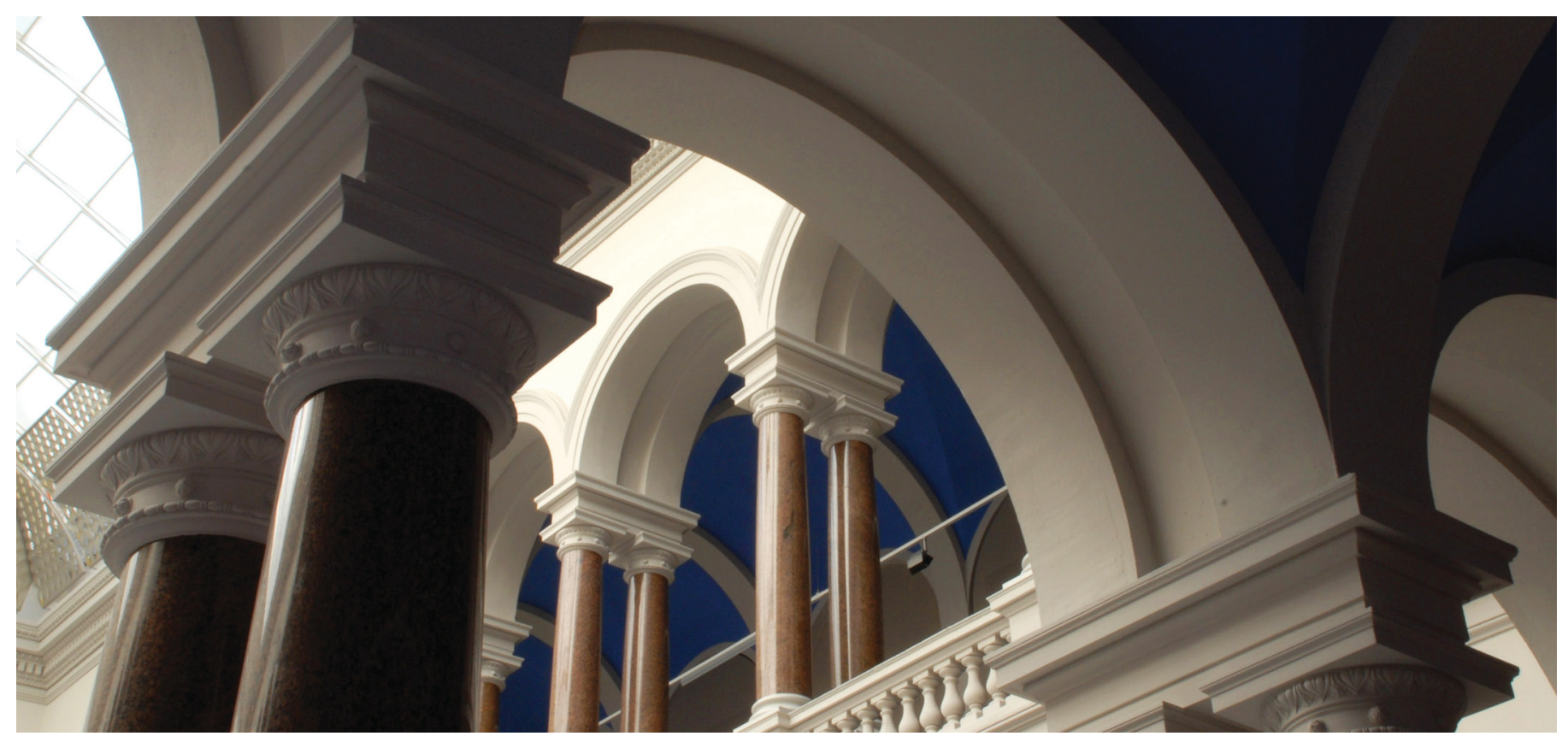

This is a post-peer-review, pre-copyedit version of an article published in Österreichische Zeitschrift für Soziologie. The final authenticated version is available online at: http://dx.doi.org/10.1007/s11614-018-0317-7

Ullrich, P. (2018). Videoüberwachung von Demonstrationen und die Definitionsmacht der Polizei. Zwischen Objektivitätsfiktion und selektiver Sanktionierung. Österreichische Zeitschrift für Soziologie, 43 (4), 323-346. https://doi.org/10.1007/s11614-018-0317-7 


\title{
Videoüberwachung von Demonstrationen und die Definitionsmacht der Polizei. Zwischen Objektivitätsfiktion und selektiver Sanktionierung
}

\begin{abstract}
Untersucht wird Videoüberwachung im Protest Policing auf Basis von Gruppendiskussionen und Expert/inneninterviews mit Polizist/innen sowie ethnografischen Beobachtungen. Die Polizei legitimiert Videoüberwachung mit dem Versprechen von Objektivität und strikter Rechtsdetermination. Im Aufsatz wird sie stattdessen als kontingenter Prozess der aktiven Konstruktion von Evidenz analysiert. Er besteht aus einer Abfolge von Entscheidungen in drei Grundphasen: von der Potenzialbestimmung über die polizeilich orientierte Durchführung zur staatsanwaltschaftlich orientierten und auf Verurteilung abzielenden Fixierung der Prozessergebnisse in der Nachbereitungsphase. Die Breite der Handlungsoptionen bis hin zur Manipulation wird als Ausdruck soziologischen Ermessens begriffen, in welchem die polizeiliche Definitionsmacht gründet. Insbesondere die beteiligte Sachtechnik ermöglicht, dass die bei jedem Teilschritt bestehende Kontingenz im weiteren Verlauf über Objektivationen und Abstraktionen unsichtbar gemacht wird. Das Recht erweist sich in dieser Definitionsmachtkette als nur ein Handlungsmotiv unter vielen, die Rechtsdeterminiertheit des Polizeihandelns als notwendige Fiktion.
\end{abstract}

Schlüsselwörter: Soziale Bewegungen, Protest, Demonstrationen, Polizei, Videoüberwachung, Protest Policing, soziologisches Ermessen, Definitionsmacht

\section{Einleitung $^{1}$}

Videoüberwachung zur „Beweissicherung und Dokumentation “2 gehört für Demonstrierende zum Protestalltag. Auseinandersetzungen um die Rechtmäßigkeit polizeilichen Filmens bremsten die Tendenz der Ausweitung etwas ab und führten zu zunehmender Regulierung; die Polizei hatte zu oft ohne Rechtsgrundlage gefilmt. Schon das bloße Bereithalten von auf Demonstrierende gerichteten Kameras ist nach herrschender Rechtsauslegung ein Grundrechtseingriff. Die durch den juristischen Diskurs geprägte öffentliche Auseinandersetzung thematisiert insbesondere die möglichen Abschreckungseffekte. Mit dem Fokus auf Gefahren für Versammlungsfreiheit und informationelle Selbstbestimmung ist sie gewissermaßen ,betroffenenzentriert'. Die Polizei und die Hintergründe ihres Handelns sind damit aus dem Fokus gerückt. Doch welchen Logiken folgt der Videoeinsatz? Warum, wie und wen filmt die Polizei? Gibt es systematische Selektivitäten? Gerade angesichts der

\footnotetext{
${ }^{1}$ Entstanden im DFG-Projekt „Videoüberwachung von Versammlungen und Demonstrationen“ (ViDemo, GZ: UL 389/3-1). Ich danke A. Jacobsen, P. Knopp und D. Hunold für Hinweise.

2 Bildübertragung ohne Aufzeichnung wird hier nicht behandelt.
} 
Fälle rechtswidrigen Filmens stellt sich die Frage, welche Motive neben dem oder gar anstelle des Rechts handlungsleitend sind und die Überwachungspraxis konstituieren.

Während sich - wie zu zeigen sein wird - polizeiliche Begründungsmuster und Legitimationsargumentationen beim Thema Videoüberwachung normativ am Ideal der Rechtsprogrammierung des Polizeihandelns und substanziell an der Fiktion einer durch die Überwachungsvideos sichergestellten dokumentarischen Objektivität orientieren, offenbart der Blick in die Praxis die Kontingenzen polizeilicher Videodokumentation. Polizeiarbeit ist geprägt durch einen „nahezu uferlosen Ermessensspielraum“ (Backes, Dollase und Heitmeyer 1998, S. 5). Der Spielraum dieses nicht nur juristischen, sondern soziologischen (oder ,tatsächlichen') Ermessens und das Spannungsverhältnis zwischen beiden soll für die Videoüberwachung von Demonstrationen untersucht werden. Dazu wird in techniksoziologischer Perspektive analysiert, welche Rolle Handlungsroutinen und genutzte Sachtechnik (vgl. Schulz-Schaeffer 1999) bei der Konstruktion von Evidenz spielen. Dafür muss ein analytischer Beschreibungsrahmen entwickelt werden, der Videoüberwachung als Prozess rekonstruierbar macht. Unter Videoüberwachung wird hier entsprechend nicht nur der jeweilige konkrete Akt des Filmens verstanden, sondern das gesamte auf die Beweis- und Dokumentationsaufnahmen bezogene Arrangement bis zur Nutzung der Bilder.

Im Folgenden wird der Forschungsstand (2) und das methodische Vorgehen erläutert (3). Im Ergebnisteil wird zunächst dargestellt, wie Videoüberwachung polizeiseitig begründet wird (4). Der folgende Teil widmet sich der Rekonstruktion eines idealtypischen Verlaufs von Videoüberwachung als Prozess, in dem über Schritte von Objektivierungen (die polizeiliche Definitionsmachtkette) eine partikularistische Sichtweise auf ein konflikthaftes Interaktionsgeschehen den Status von Objektivität zugeschrieben bekommt (5). Dann werden Faktoren herausgearbeitet, die die Chance, in den Kamerafokus zu gelangen, begünstigen (6). Im Fazit wird reflektiert, welche Bedeutungen der analysierte Prozess im institutionellen Zusammenhang für Ungleichheiten in der politischen Partizipation hat.

\section{Forschungstand}

\subsection{Videoüberwachung und selektives Protest Policing}

Der Forschungsstand zum Thema ist übersichtlich. ${ }^{3}$ Es gibt polizeiliche Literatur, die sich vorrangig mit polizeipraktischen Fragen befasst (bspw. Timmermanns 2010). Umfangreicher ist die juristische Literatur, die u.a. als Reflexion der gerichtlichen Auseinandersetzungen ${ }^{4}$ und neuer Versammlungsgesetze auf Länderebene entstand (Koranyi und Singelnstein 2011; Kutscha 2011; Arzt und Ullrich 2016). Hier wird überwiegend das Spannungsverhältnis zwischen der Videoüberwachung und der grundgesetzlich geschützten Versammlungsfreiheit diskutiert und damit die Eingriffsschranken für Polizeihandeln. So darf Videoaufzeichnung nur zur Verfolgung konkreter Straftaten (nach der Strafprozessordnung) und (nach dem Versammlungsrecht) bei Vorliegen tatsächlicher Anhaltspunkte (nicht auf bloßen Verdacht) für erhebliche Gefahren für die öffentliche Sicherheit oder Ordnung (also nicht bspw. bei Ordnungswidrigkeiten) zum Einsatz kommen.

Die sozialwissenschaftliche Literatur behandelt Videoaufzeichnungen in allgemeinen Darstellungen zum Protest Policing nur en passant neben anderen Einsatzmitteln und -strategien. Sie ist Teil der technischen Überwachungsmaßnahmen, die im Zuge der gegenwärtigen Militarisierung des Protest Policing (Wood 2014) an Bedeutung gewinnen. Liberale Entwicklungen des Protest Policings seit den siebziger Jahren des vergangenen Jahrhunderts (McPhail, Schweingruber und McCarthy 1998) mit

\footnotetext{
${ }^{3}$ Zum Überblick vgl. Ullrich (2014); zur Videoforensik Tuma (2017, 129 ff.).

${ }^{4}$ Ullrich (2014, 42 f.), VG Hannover, 10 A 226/13, VG Leipzig, 1K 222/13 \& 1K 259/12.
} 
dominant deeskalativer Orientierung (negotiated management) werden seit der Auseinandersetzung mit den Protestformen der Globalisierungskritik durch den Ansatz der strategic incapacitation (Gillham und Noakes 2007) ergänzt oder abgelöst. Der neue Policing-Stil fußt auf den drei Säulen Überwachung/Information, proaktives Policing und Raumkontrolle (Gillham und Noakes 2007, 349 ff.). Diese Strategien dienen einem selektiven Zugriff auf Protestierendengruppen, welche durch binäre Zuschreibung von Legitimität/Nichtlegitimität in Normalitätsklassen eingeordnet werden (Ullrich 2017). Ullrich und Wollinger (2011) deuten Videoüberwachung entsprechend als Hilfsmittel dieser symbolischen Sortierung von ,guten' (nichtüberwacht) und ,bösen' (durch Kameraüberwachung markiert) Demonstrierenden. Zu der Positionszuweisung für Protestierende kommen weitere Funktionen für die Kontrolle von Protesten. Videoüberwachung ist sowohl situatives panoptisches Disziplinierungsarrangement für die von ihr direkt betroffenen als auch abstrakte Anrufung (im Sinne der Gouvernementalitätstheorie) an alle Demonstrierenden, sich zur Überwachung, derer man nie ganz gewiss sein kann, in ein reflexives Verhältnis zu setzen (ebd.).

Aus der Perspektive der Surveillance Studies betonen Ullrich/Wollinger auch die Einbindung der Videoüberwachung in die surveillant assemblage (Haggerty und Ericson 2000). Mit dem Assemblagebegriff wird die Dezentralität, also die umfassende, heterogene, multilokale, kurz: rhizomatische Struktur gegenwärtiger Überwachungsgesellschaften thematisch. Deren Entstehung fußt auf äußerst heterogenen desires nach Daten. Auch die in Reaktion auf polizeiliche Überwachung entstehende Gegenüberwachung seitens Protestierender (u.a. durch Handyvideos) ist, weil sie ebenso Daten produziert, Teil der assemblage. Zudem beobachten sich die konfliktären Akteure, um innovativ auf die Taktiken der anderen zu reagieren (Ullrich 2014, S. 46; Knopp und Ullrich 2016), wodurch sie eine Spirale von Überwachung und Gegenüberwachung konstituieren (D. J. Wilson und Serisier 2010).

Eklatante Forschungslücken bestehen hinsichtlich der konkreten Auswirkungen auf Demonstrierende (explorativ dazu Ullrich 2011, 39 ff.) und der organisationalen Motive und Praxislogiken ihres Einsatzes, also der Konstruktion von Evidenz mittels der Videoforensik (Tuma 2017, 130-33). Ausschließlich der zweite Themenkomplex wird hier verfolgt.

\subsection{Soziologisches Ermessen und polizeiliche Definitionsmacht}

Die dokumentierten devianten Polizeipraxen werfen die soziologisch interessante Frage auf, inwiefern polizeiliche Praxis unabhängig von den Freiheitsgraden des rechtlichen Ermessens einem größeren faktischen oder „soziologischen“ (Brusten 1971, S. 34; vgl. a. Skolnick 1966, S. 71 ff.) Ermessen unterliegt.

Das soziologische Ermessen, ein Begriff, den die ethnographische Polizeiforschung der siebziger Jahre geprägt hat, umfasst die gesamte Breite tatsächlich realisierbarer Handlungsoptionen der Polizei. Es resultiert strukturell daraus, dass

- universalistische Rechtsnormen situational unterbestimmt sind, also keine konkreten Handlungsanweisungen beinhalten und situationsbezogene Deutungen erforderlich machen; ${ }^{5}$

- ein Großteil von Polizeihandeln sich ohnehin im vorrechtlichen Bereich von Alltagskonflikten vollzieht und

- dass Polizei immer auch Moralakteur ist, der in der Ausführung des Auftrags, Recht und Ordnung zu schützen, diese (insbesondere die nicht definierbare „Ordnung“) auch immer wieder aktiv und in Auseinandersetzung mit sich schneller als das Recht wandelnden herrschenden Moralvorstellungen konstruieren muss (vgl. Hunold 2015, 9 ff.).

\footnotetext{
${ }^{5}$ Zur Prekarität der Anwendung formaler Regeln in Organisationen vgl. Luhmann (1976, 309 f.).
} 
Daher sind „Ermessensentscheidungen als konstitutives Merkmal polizeilicher Praxis zu begreifen [...] und unausweichliche Voraussetzungen, um überhaupt handlungsfähig zu sein" (Hunold 2015, S. 11). Erwartbar ist dabei durchaus auch ein Anteil illegalen Handelns, teilweise in Form von aus Organisationssicht „brauchbare[r] Illegalität“ (Luhmann 1976, S. 304 ff.), die entsteht, wenn eine widerspruchsfreie Normordnung mit Umwelten kollidiert, die dieser Widerspruchsfreiheit entgegenstehen. Daher erfordert sie das Latenthalten ihrer Brauchbarkeit und entsprechende Schutzhandlungen. Aus dieser Funktion resultieren u.a. Widersprüche zwischen Legitimierungserzählungen und Praxisberichten.

Die strukturelle Notwendigkeit, Ermessensentscheidungen zu treffen, führt in der Anwendung daher zu ihrer Prägung durch Handlungsmotive der Anwendenden bzw. der Organisation und damit zu einer spezifischen Selektivität. Die klassischen Studien dazu interessierten sich insbesondere für Sanktionierungsverhalten von Streifenpolizist/innen. Ausgangspunkt war die Beobachtung einer „Normaltendenz" von underpolicing (J. Q. Wilson 1968, S. 173), die u.a. daraus folgt, dass eine Polizei mit begrenzten Ressourcen trotz Strafverfolgungszwang faktisch nicht in der Lage ist, alle Normverstöße zu verfolgen. Welche systematischen Gründe zu realisiertem Verdacht und Sanktionsverhalten führen, ist Gegenstand der Forschung zu den „Determinanten selektiver Sanktionierung" (Brusten 1971), die Brusten in Strategien der Erfolgsmaximierung, der individuellen Aufstiegsorientierung und der polizeilichen Perzeption der Bewertung ihres Handelns durch Gerichte vermutet. Feest und Blankenburg (1972) arbeiteten zudem heraus, dass tatsächliche Strafverfolgung von Stigmata, der bipolaren Unterscheidung „anständig/kriminell“, der sozialen Distanz zu bestimmten Gruppen und der Eigenaktivität/dem Anzeigeverhalten abhängt. Die systematische Selektivität des Sanktionierungsverhaltens zeigt sich u.a. in schichtspezifischen Kriminalisierungsraten (Brusten 1971). In dieser spezifischen Rolle der Polizei als Inhaberin des staatlichen Gewaltmonopols mit Ordnungs- und Strafverfolgungskompetenzen, die für solche Entscheidungen verantwortlich zeichnet, gründet also die Entscheidungsmacht (Jasch) oder Definitionsmacht der Polizei (Feest/Blankenburg), ihre „sozial vorstrukturierte Chance, eine Situation für andere verbindlich zu definieren" (Feest und Blankenburg 1972, S. 19; Jasch 2002, S. 78 ff.).

Diese Macht nutzten Polizist/innen beispielsweise, um als unangemessen empfundene Unbotmäßigkeit zu sanktionieren. Dies spielt im oft konfliktgeladenen Protest Policing wahrscheinlich eine große Rolle. Denn die Herstellung und Sicherung von Autorität und situativer Dominanz ist bestimmendes Motiv von Polizeihandeln (Feest und Blankenburg 1972, S. 70; Loftus 2010; Fekjær, Petersson und Thomassen 2014), wird aber bei Demonstrationen oft herausgefordert (Greer und McLaughlin 2010; Ullrich 2017). In der Forschung über Protest Policing wurde bisher nicht explizit mit dem soziologischen Ermessensbegriff gearbeitet, wenngleich sich vorliegende Literatur in diese Richtung „übersetzen“ lässt (s.u.). Was die Klassiker primär auf Sanktionierung von konkreten Personen mit Polizeikontakt beziehen, muss als Rahmen einer Analyse von Sanktionshandeln im Protest Policing in zweifacher Hinsicht erweitert werden: Zum einen sind die von Videoüberwachung tangierten nicht nur Individuen, sondern i.d.R. Gruppen oder Menschenmengen. Zum zweiten muss der medial turn in Rechnung gestellt werden und auch dies in doppelter Hinsicht. Einerseits ist Medienvermitteltheit offensichtlich basales Merkmal des analysierten Film-Prozesses: als „skopisches Medium“ macht es durch „Beobachtungs- und Bildschirmtechnologien [...] distante bzw. unsichtbare Phänomene situational präsent", kann sie wie eine Linse brechen, bündeln, fokussieren oder projizieren (Knorr-Cetina 2012, S. 168, 170). Andererseits hat eine selektive Sanktionierungspraxis Folgen nicht nur für konkrete Personen in eng umgrenzten Situationen, sondern zugleich im Hinblick auf das veröffentlichte Bild von Protesten und damit von bestimmten politischen Strömungen (Schmitt-Beck 1990). Der Polizei als primary definer, der in der hierarchy of credibility (Becker 1967) weit oben angesiedelt ist, kommt dabei eine herausgehobene Rolle zu. Polizeiliche Deutungen von Ereignissen werden vor Gericht wegen ihres "Glaubwürdigkeitsbonus" (Eisenberg, Voigt und Vogel 
2014, S. 92; Singelnstein 2014) wie in den Medien (Dießelmann 2015, bes. S. 123) präferiert, wenn nicht eine hohe gegenläufige Skandalisierungsschwelle überwunden wird (Greer und McLaughlin 2010).

Von medialer Präsenz hängt die Ermessensausschöpfung im Protest Policing tatsächlich ab. Soziologisches Ermessen zeigt sich konkret in der Wahl von policing styles im Umgang mit bestimmten Demonstrierendentypen. Neben einer Vielzahl eher tentativer Einschätzungen (u.a. Waddington 2007, S. 35; Gillham und Noakes 2007; Wood 2014), kommen auch die wenigen systematischen Untersuchungen (Earl, Soule und McCarthy 2003; Wisler und Giugni 1999) zu einem ähnlichen Ergebnis: härteres Policing ist bei marginalisierten, mithin weniger beschwerdemächtigen, und antagonistischen (oder ,radikalen') Demonstrierendengruppen wahrscheinlicher (vgl. a. Ullrich 2017). Entsprechend stellt sich die Frage, ob für Entscheidungen in Bezug auf Videoüberwachung ebenso diese beiden Faktoren und gegebenenfalls welche weiteren für under/over-enforcement bzw. under-/over-watching verantwortlich sind. Welcher Handlungsspielraum besteht konkret (ggf. in Abhängigkeit vom Überwachungsobjekt), Bilder aufzuzeichnen oder nicht, sie zu speichern, löschen, bearbeiten und sonstwie (nicht) zu verwenden?

\section{Methoden}

Polizeiliche Videoüberwachung, ihre Wirkungen auf Protestierende und die (medial vermittelten) Bezugnahmen beider Seiten aufeinander wurden im Projekt "ViDemo“ in einem Grounded-TheoryDesign (Strauss und Corbin 1998) untersucht. Quellen waren Gruppendiskussionen mit Polizist/innen und Demonstrierenden in drei deutschen Bundesländern, Expert/inneninterviews, teilnehmende Beobachtungen auf Demonstrationen und Dokumente wie Einsatzleitlinien, Gerichtsurteile usw. (Erhebungszeitraum v.a. 2014-2016). Im Fokus der Prozessanalysen hier stehen: ${ }^{6}$

1) Elf Gruppendiskussionen mit je etwa vier Beamt/innen, überwiegend aus Einsatzhundertschaften der Bereitschaftspolizei, meist sogenannte „Bedo“-Beamt/innen” (auch Bedo-Ausbilder/innen, Lehrgangsteilnehmer/innen, Mitglieder von Technischen Einsatzeinheiten, Beweissicherungs- und Festnahmeeinheiten).

2) Elf Expert/inneninterviews, v.a. mit Vorgesetzten mit Verantwortung für Protest Policing (Hundertschaftsführer/innen, Einsatzführer/innen, Justiziar/innen, Stabsmitarbeiter/innen) sowie einem Vertreter eine Polizeigewerkschaft und einem Hochschullehrer für Einsatzlehre.

Es dominieren also Selbstzeugnisse. Gruppendiskussionen wurden gewählt, um den

Gruppencharakter der Einsatzsituation und damit Sinnkonstruktionen der Gruppen und der durch sie repräsentierten Organisation rekonstruierbar zu machen. ${ }^{8}$ Es wird also davon ausgegangen, dass die Befragten sozial erwünschte Antworten geben, die sich an den Normen der Polizeikultur orientieren, also an einem durch Recht und Leitlinien geprägten Deutungsangebot der Organisation und ihrer Funktionseliten an ihre Mitglieder und die Gesellschaft (Behr 2006; i.e. „Schauseite“ der Organisation, Kühl 2014, S. 333). Diese Schauseitenpräsentation wird aber, wie die Gesprächsverläufe zeigen, immer wieder von individuellen und Gruppenmechanismen überlagert.

\footnotetext{
${ }^{6}$ Die Dokumente sind durch eine Nummer plus Code zum Dokumenttyp gekennzeichnet ( $G D=$ Gruppendiskussion, INT=Expert/inneninterview, $F P=F e l d p r o t o k o l l)$. Betonungen sind fett gedruckt, Unverständliches in doppelten Klammern, Abbrüche sind durch „,“" gekennzeichnet. Zitate wurden sprachlich behutsam geglättet.

7 „Bedo"/„Besi“ - Polizeiakronyme für „Beweissicherung und Dokumentation“; „Bedos“ im Folgenden auch kurz für Bedo-Beamt/innen.

${ }^{8}$ Als Stimulus wurde i.d.R. eine Videosequenz aus dem Versammlungsgeschehen mit sichtbaren Kameras gezeigt. Die Teilnehmenden wurden dann um eine Interpretation und Deutung aus Sicht ihrer eigenen Arbeit im Feld gebeten und sollten selbstläufig diskutieren.
} 
Erzählzwänge (Aufschaukeln, narrative Triftigkeit, Selbstläufigkeit) werden in Gang gesetzt und überwinden so gelegentlich gewisse Artikulationshemmungen, v.a. bei der Besprechung von unangenehm empfunden Themen oder illegalen Verhaltensweisen und gewähren Einblicke in die Praxis. Die Koexistenz dieser beiden Ebenen wird in der Inkonsistenz zwischen verschiedenen Antwortmodi (Praxisdeutung vs. Legitimationserzählungen) sichtbar. ${ }^{9}$

Die grundsätzliche schwierige Zugänglichkeit der Polizei für Forschung durch einen hohen Grad organisationaler Schließung sowie das häufige Bemühen, steuernd in den Prozess einzugreifen, müssen als Gültigkeitseinschränkungen thematisiert werden. Die starke polizeiliche Gatekeeperrolle hatte Kompromisse u.a. im Sampling zur Folge (ausführlich in Ullrich 2018). Dies kann durch Triangulation mit anderen Daten zumindest teilweise aufgefangen werden, darunter ethnografische Beobachtungen auf Demonstrationen (über 40 Feldprotokolle), drei Expert/inneninterviews mit Beobachter/innen des Versammlungsgeschehens, ergänzende Hintergrundgespräche sowie Dokumentenanalysen.

Alle mündlichen Quellen wurden verschriftlicht, anonymisiert und zunächst offen (,entdeckend') kodiert. Dabei stehen additive Momente des möglichst breiten Sammelns von Informationen über einen weitgehend unerforschten Gegenstand neben dem zur theoretischen Verdichtung entscheidenden kontrastiven Vorgehen beim axialen Kodieren im Hinblick auf die sich herausschälende Kernkategorie der Definitionsmachtkette.

\section{Motive - polizeiliche Erwartungen an Videoüberwachung}

Den zentralen Hintergrund für den vermehrten Einsatz von Videotechnik bei Protestereignissen stellt das lange bestehende Missverhältnis zwischen der Anzahl verhafteter Verdächtiger und tatsächlichen Verurteilungen dar. Die Videotechnik soll eine „lückenlose Beweiskette“ (INT_25) sicherstellen, die gerichtlichen Evidenzanforderungen gerecht wird. Das organisationale Motiv der Beweissicherung als antizipierte Erwartung von Staatsanwaltschaften und Gerichten dominiert auch die Darstellungen im empirischen Material. Es ermöglicht zudem Beweissicherung ohne unmittelbaren Handlungszwang; die Festnahme kann auch zu einem späteren Zeitpunkt stattfinden. Aus Sicht der Beamten verbindet sich dies noch mit Sekundärnutzen: das dokumentieren einer Straftat dient als Erfolgsnachweis und es kann Strafbedürfnisse befriedigen, z.B. wenn versucht wird, einen Demonstrierenden dessen mutmaßlichen Gesetzesbruch man nicht rechtzeitig aufzeichnen konnte, mithilfe verstärkten Filmens später noch bei einer anderen Tat ,zu erwischen' (052_GD, vgl. a. Tuma 2017, S. 139).

Schon die Bezeichnung der entsprechenden polizeilichen Expert/innen benennt ein zweites organisationales Motiv: die Dokumentation des eigenen Vorgehens. Dieser Aspekt wird in den Gesprächsprotokollen v.a. als Absicherung gegen , unberechtigte' Vorwürfe thematisiert. Auch die Dokumentation polizeilichen Fehlverhaltens wird - allerdings immer in individualisierender Zuschreibung an „Schwarze Schafe“ (vgl. a. Behr 2000b) - erwähnt, allerdings im Gegensatz zu Rechtsbrüchen des Gegenübers als seltene Ausnahme nicht detailliert. Rechtlich ist das Absicherungsmotiv problematisch, da es durch Eingriffsvoraussetzungen nicht erfasst ist. Aufschlussreich ist in diesem Zusammenhang, dass eine Arbeitsmappe einer Landespolizei mit Leitlinien für Bedo-Beamte entsprechend auch die „Imageverbesserung der Polizei“ als Ziel aufführt.

Das dritte Motiv, welches sich indirekt auch aus Wirkungszuschreibungen an die Kameras erschließt, ist Prävention, auch wenn es über diesbezügliche Erfolge keineswegs Einigkeit unter den Befragten

\footnotetext{
${ }^{9}$ So in mehreren Gruppendiskussionen, in denen einerseits argumentiert wird, dass Videokameras für niemanden eine Beeinträchtigung darstellten, während an anderer Stelle kamerainduzierte Aggressionen und Verhaltenssteuerungseffekte thematisiert werden.
} 
gibt. Auch hier beziehen sich die Argumentationen, mit einer Ausnahme, auf Prävention unerwünschten Verhaltens von Demonstrierenden. Beschrieben werden v.a. vermeintliche Tatvorbereitungssituationen wie das Aufnehmen von Steinen oder das Anlegen von Vermummung, die durch Sichtbarkeit oder intentionales Zeigen der Kameras unterbrochen worden seien. Eine mögliche Abschreckung von der Teilnahme an Demonstrationen durch Videoüberwachung wird zumindest als Möglichkeit erwogen, meist jedoch mit dem Verweis auf Rechtsstaatlichkeit, Neutralität und den sowieso i.d.R. sparsam erfolgenden Einsatz von Filmaufnahmen zurückgewiesen. Ohnehin verlaufe alles - wie immer wieder betont wird - rechtmäßig. Kondensiert kommt dies im in den Gruppendiskussionen wie Interviews immer wieder bemühten klassischen Argument der Kritiker/innen von Überwachungskritik zum Ausdruck, dass nämlich Überwachung diejenigen nicht störe, die sich nichts zuschulden kommen ließen.

Damit wird Kritik an Überwachung völlig verengt in den legalistischen Deutungshorizont der Polizei überführt. Zudem wird die grundsätzliche Logik der Legitimierungserzählung von Videoüberwachung verdeutlicht, die vor allem auf einem mit ihr verbundenen dokumentarischen Objektivitätsideal beruht. Dieses baut auf die „argumentative Kraft“ (Kammerer 2008, S. 52), nicht zuletzt im Hinblick auf die Täter selbst, die schon immer Motor der Einführung von Videoüberwachung auch in anderen Bereichen war (ebd.). Die Bilder würden, so betonen viele Interviewte, „ein Stück weit Objektivität“ (022_GD) sicherstellen. Sie werden überwiegend als neutrale Fakten begriffen, die somit gleichsam interesselos im Dienst der Wahrheit stünden. Kritik wird damit von vornherein als ungerechtfertigt zurückgewiesen. Denn, um

„auszuschließen, ... dass man halt wirklich mal den Falschen sich greift, ist es, denke ich mal, auch für beide Seiten gut." (022_GD)

Um erfolgreiche Prävention und Strafverfolgung unter diesen postulierten hohen Idealen Rechtsgesteuertheit, Neutralität, Objektivität, Datensparsamkeit - sicherzustellen, verfügen alle Polizeien über entsprechende ausgebildete Spezialist/innen (i.d.R. zweiwöchiger Lehrgang, Fortbildungen und ggf. Lehrgang zum Bedo-Trupp-Führer). Manche Befragte waren aber auch schon vor der Absolvierung des Lehrgangs entsprechend tätig. Man kann diesen Widerspruch zwischen Ideal und Realität als produktiven Zweifel aufgreifen, auf den gesamten Prozess übertragen und fragen, in welchem Verhältnis die in legitimatorischer Absicht formulierten Ideale zu den Möglichkeiten, Zwängen und praktischen Routinen im Einsatz stehen.

Nachrangige Motive für Bildanfertigung und -nutzung sind die Auffrischung des Gedächtnisses vor gerichtlichen Zeugenaussagen und die Nutzung von Filmen zur Einsatznachbesprechung oder für Ausbildungszwecke.

\section{Die Definitionsmachtkette - polizeiliche Videoüberwachung als Prozess}

Videoüberwachung wird im Folgenden als idealtypischer Verlauf aufeinander aufbauender Handlungsoptionen und Entscheidungen rekonstruiert. Idealtypisch ist diese Prozessdarstellung, da nicht alle Schritte immer und nicht immer starr in dieser Reihenfolge stattfinden, es auch Rekurse, Wiederbeginn oder Abbrüche gibt und die Praxen sich zwischen Bundesländern und Einheiten unterscheiden, ohne dass dadurch die temporale Grundlogik in Frage gestellt wäre. Der Prozess lässt sich in die drei Grundphasen Vorbereitung, Durchführung und Nachbereitung mit jeweiligen Teilschritten gliedern (Abb. 1).

In diesem Analyserahmen ist für jeden Teilschritt herauszuarbeiten, welche Ermessensspielräume sich ergeben, also wie kontingent die Entscheidungen sind, und welche Anschlusslogiken mit getroffenen Entscheidungen jeweils produziert werden. 


\subsection{Vorbereitung: Potenzialbestimmung}

Die „Protestdiagnose“ (Winter 1998a) über zu erwartende Verläufe und Gefahrenpotenziale steht am Anfang des Prozesses und erfolgt auf Basis von allgemeinen Erfahrungen, Erfahrungen mit beteiligten Akteuren, durch Recherchen in internen Informationssystemen und öffentlichen Quellen oder durch Konsultation von "szenekundigen Beamten“ und resultiert bei ausreichender Vorbereitungszeit in einem schriftlichen Einsatzbefehl. Für Bedo-Beamt/innen kann eine weitere Vorbereitung auch darin bestehen, sich detaillierter über das erwartete „Klientel“ zu informieren, also bspw. über typische verbotene Symbole, bekannte "Gefährder" u.ä. Vor dem Einsatz wird das Video mit Kontextinformationen (Anlass, Ort, Zeit usw.) besprochen.

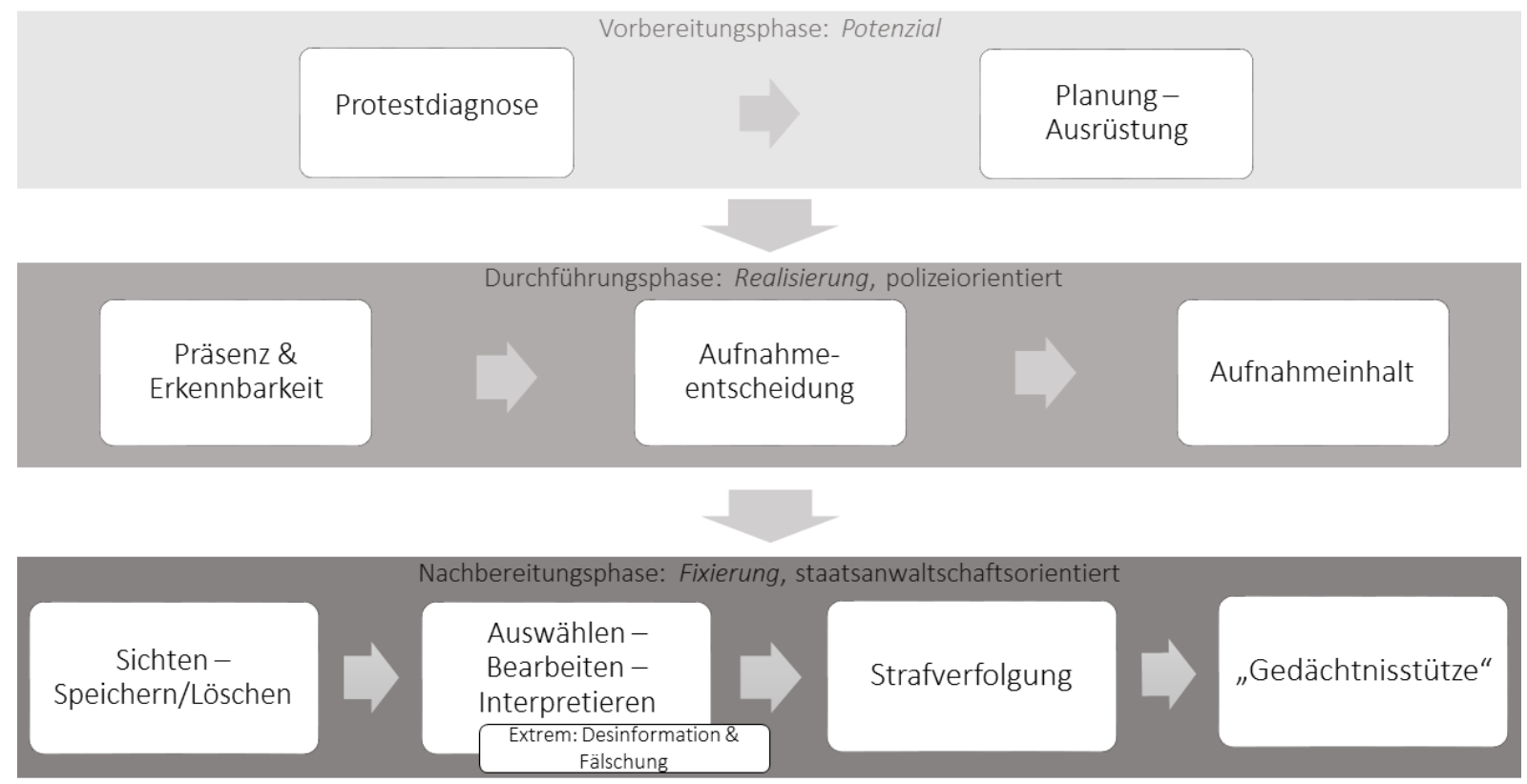

Abb. 1 Phasen, Teilschritte und Orientierungsinstanzen im Prozess polizeilicher Videoüberwachung von Demonstrationen

Dass binär codierte Stereotype und starke Feindbilder die polizeilichen Gefahreneinschätzungen prägen, ist ausführlich gezeigt worden (u.a. Willems u. a. 1988; Winter 1998a; Ullrich 2017). Zu beachten ist hier, dass in die in Demonstrierendenklassifikationen verbreitete Grundunterscheidung zwischen als legitim erachteten "Normalbürgern“ oder "Normaldemonstranten“ und den feindlich perzipierten „Störern“, „Chaoten“ oder „Krawalltouristen“ auch inhaltliche Bewertungskriterien eingehen, d.h. dass Legitimitätszuschreibungen in Legalitätsbewertungen überführt werden. Neben juristisch legitimierten Kriterien wie tatsachenbasierter Gewalterwartung gehören zugeschriebene Gruppenzugehörigkeiten, äußerliche Erscheinungsmerkmale und Bewertungen politischer Anliegen zu den relevanten Gefahrensignalen (Ullrich 2017; Arzt und Ullrich 2016). Die Protestdiagnose bestimmt weitgehend die Einsatzplanung (Eggert u. a. 2016) und damit auch, ob und in welchem Maß Videotechnik vorgehalten wird. Bei der Mehrzahl kleinerer Versammlungen gibt es keine oder minimale Polizeipräsenz. Ist Bereitschaftspolizei anwesend, ist damit i.d.R. auch Videotechnik vor Ort. Die Größe der Veranstaltung und politischer Antagonismus der Protestakteure sind für die Inzidenz polizeilichen Filmens entscheidende Faktoren (Knopp und Müller-Späth 2017).

Eine stereotypenbeeinflusste Protestdiagnose bestimmt also Ausrüstung und Ausstattung mit und damit die (ungleich verteilte) Chance, überhaupt videografiert werden zu können; hier entscheidet sich das Überwachungspotenzial. 


\subsection{Durchführung: Bildproduktion}

Sind nun Kameras vor Ort, ist eine Vielzahl weiterer Entscheidungen über die Realisierung des Potenzials überhaupt erst möglich.

\section{Präsenz und Erkennbarkeit}

Kameras können unterschiedlich vorgehalten werden: als Mastkamerawagen (BeDoKW), als Handkamera (meist mit Teleskopstab) griffbereit im Fahrzeug oder gleich mit Beamt/innen sichtbar um die Demonstration herum verteilt. Dazu gibt es bei einschließender Begleitung oft im Abstand von einigen Metern jeweils eine/n Bedo. Teilweise werden erhöhte Stadtorte gesucht, die Übersicht gewähren und die Bedos zum „Auge des Zugführers [...] auf der Suche nach Ordnungswidrigkeiten und Straftaten" (034_GD) machen. Dass die Entscheidung, Kameras sichtbar vorzuhalten, Effekte auf die Demonstrierenden hat, bestätigen nicht nur die Gruppendiskussionen mit Aktiven, sondern auch die polizeilichen Berichte über häufige kritische/negative Reaktionen auf die Anwesenheit von Kameras. Dazu gehören Taktiken des Verbergens und Sich-Entziehens, der argumentativen Auseinandersetzung bis hin zur Beleidigung oder zum Angriff auf die Kamera(träger/innen).

Ob gefilmt wird oder nicht, ist für Demonstrierende nicht immer kenntlich. Tatsächliches Filmen kann, aber muss nicht, über Soziale Medien, Lautsprecherwagen oder Kontakt zur Versammlungsleitung kommuniziert werden. Oft hält sich die Polizei bedeckt. Auf Auskunftsersuchen wird meist abweisend reagiert, sie werden eher als rein strategisch motivierte Störung der eigenen Arbeit begriffen (vgl. a. Ullrich 2017). Sogar explizite Falschauskünfte sind dokumentiert (siehe 5.3.2). Auch die Kamerahaltung ist hierfür relevant. Angesichts versammlungsfreundlicher Rechtsprechung setzt sich mehr und mehr durch, dass nicht filmende Kameras erkennbar nach unten gehalten werden müssen. Zum Beweis der „Ungefährlichkeit“ des Videografierens wird insbesondere von Vorgesetzten immer wieder auf diesen Umstand hingewiesen. Es gibt entsprechende Dienstanweisungen oder Standardtexte für Einsatzbefehle in liberaleren Bundesländern. ${ }^{10}$ Unsere Demonstrationsbeobachtungen (Knopp und Müller-Späth 2017; Arzt und Ullrich 2016) machten jedoch deutlich, dass dies in der Praxis nicht eingehalten wird. Oft werden Kameras ohne erkennbare Fokussierung aufrecht bereitgehalten, u.a. weil das Ausfahren der Stative "ein bisschen lästig" sein kann (075_INT). Für Außenstehende kann sich das auch als generelles Filmen der Demonstrationen darstellen oder Unsicherheit darüber verstärken.

Sowohl die Demonstrationsbeobachtungen als auch die o.a. Berichte über Einsatzmotive verdeutlichen, dass Kameras auch zur Einschüchterung verwendet werden können. Dies wird besonders in einer Szene am Rande einer Antifa-Demonstration deutlich (001_FP, ausführlich Knopp und Müller-Späth 2017, S. 12). Ohne konkrete Gefahren wurde die Kamera fast spielerisch zur Machtdemonstration verwendet, hier augenscheinlich, um missliebige Beobachtung der polizeilichen Tätigkeit zu erschweren (vgl. Ullrich 2018).

Anschlussfragen für den Prozess sind nun, ob und wenn ja, was tatsächlich aufgezeichnet wird.

\section{Aufnahmeentscheidung}

Ob die Eingriffsvoraussetzungen gegeben sind, muss häufig ad hoc entschieden werden. Konkrete Rechtsbrüche (bspw. das Zeigen verfassungsfeindlicher Symbole) oder deutlich tatvorbereitende Handlungen (das Aufnehmen von Steinen) sind für die Bedos eindeutige Anlässe; doch viele Signale haben eher Hinweischarakter, können tatvorbereitend oder völlig gewöhnliche und legale Alltagspraxen sein. Genannt wurden hier v.a. auf Kleidung bezogene Auslöser (Schal: Vermummung oder witterungsangemessene Kleidung? Jackenwechsel: wegen Wetteränderung oder um

\footnotetext{
${ }^{10}$ Entsprechend werden auch Konflikte mit Unterstützungskräften aus anderen Bundesländern beschrieben, die "doch noch ein bisschen mehr Freiheiten“ haben (024_GD).
} 
Identifikation zu erschweren?) und Interpretationsunsicherheit aufgrund fehlender Sprach- oder Symbolkenntnis. Die Entscheidung über das Anschalten wird von fast allen Befragten trotzdem als grundsätzlich nicht schwierig beschrieben (nur einige jüngere Bedo-Lehrgangsteilnehmer/innen fügen sich nicht in dieses Muster und Bejahen zumindest auf Nachfrage die Existenz einer Ambivalenzzone). Handlungsleitend und die (aus rechtssoziologischer Sicht immanente) Unsicherheit überwinden helfend sind konkretes professionelles Wissen (Akteursfeldkenntnis, Symbolverständnis und Rechtskenntnisse) und v.a. etwas, was immer wieder als implizite, vorbewusste "Erfahrung" (auch Lebens-, Berufserfahrung), „Gespür“, „Feeling“, „Bauchgefühl“ für „,brenzlige Situationen“ oder "gesundes Unrechtsbewusstsein“ beschrieben wird, von einem Informanten als „innere Gefahrenprognose" (034_GD_Polizei) auf den Punkt gebracht. Wie unterbestimmt diese sein kann, soll ein Zitat aus einer Narration über einen Anschaltmoment verdeutlichen:

„[...] plötzlich sieht man, da rennen die Leute. Da rennt ein Aufzug, da rennen Polizisten von links nach rechts, da passiert eventuell irgendetwas. Und dann Kamera, schalt sie an und laufe schon mit drauf $z u . "\left(022 \_G D\right)$

Die Unsicherheit ist insbesondere dynamischen Situationen inhärent.

„ich weiß nicht ob sie das kennen, dieses Runterzählen, wo man weiß, aus der Erfahrung heraus, gleich rennen die los und werden versuchen, die Spitzenkräfte zu überrennen und wo das dann halt immer so eine Sache ist. Halten die jetzt an oder rennen sie die um? Oder also habe ich jetzt hier gleich eine Straftat, also habe ich jetzt hier was oder nicht? [...] Und manch einer wartet dann halt einen Moment länger um auf Nummer sicher zu gehen [...]. Und der andere sagt: Ne, jetzt ich bin felsenfest davon überzeugt, jetzt passiert es, also mache ich die jetzt schon an. (022_GD)

Es sind solche kritischen Momente, in denen eine Hab-Acht-Stellung ausgelöst wird. Die Situation kann sich zu einer strafrechtlich relevanten entwickeln, sich häufig jedoch auch als kurzer Moment von Unruhe ohne weiteren Belang erweisen.

Neben der automatisierten, intuitiven Anschaltentscheidung mit ihren geringen

Entscheidungsunsicherheiten gibt es Anlässe, bei denen eine bewusst reflektierte Entscheidung getroffen wird und Bedos vorsichtiger agieren und genauer überlegen, ob eine Aufzeichnung rechtlich haltbar ist (u.a. bei perzipierter Beschwerdemacht, vgl. 6).

Unabhängig von der je individuellen (Un-)Sicherheit über die Eingriffsvoraussetzungen gibt es organisationale Varianz zwischen Bundesländern und Einheiten. Ob aufgenommen wird, entscheiden Bedos teils eigenverantwortlich, teils nach Rücksprache oder bei explizitem Einsatzführervorbehalt nur auf Anweisung. Die Aufnahme kann sich auf einzelne Verdächtige beschränken; diese können aber auch als Eingriffsermächtigung für das Filmen der gesamten Demonstration interpretiert werden, da ein Vorfall die Eingriffsschwelle überwunden hat. Ein Polizeiführer gab zu Protokoll, dass in seiner Einheit ausschließlich nach Strafprozessordnung gefilmt würde (also nach Vorliegen einer Straftat/eines Anfangsverdachts). Nur „wenige Augenblicke“ Vorlauf kämen dazu. Mit dieser Rechtsauffassung wird aber repressives Recht auf den Zeitpunkt vor einer Tat ausgedehnt und die höhere Eingriffsschwelle des Versammlungsrechts unterlaufen. Ein Befragter (allerdings mittlerweile nicht mehr in der Bereitschaftspolizei tätig) berichtet vom Extremfall, dass in seiner Einheit einfach immer gefilmt wurde. ${ }^{11}$ So ist es nicht überraschend, dass die (begrenzenden) Regelungen zum

\footnotetext{
${ }^{11}$ Als Antwort auf den Bericht eines Kollegen aus dem gleichen Bundesland über restriktive Filmpraxen bei seiner Einheit berichtet ein Befragter „Wir haben uns da ... nie Gedanken gemacht, ist das jetzt erlaubt oder nicht. Die waren einfach mit dabei. ... Und bis auf die einzelnen Gruppen der Bedo-Technik runter gebrochen, ich habe das nie erlebt, dass einer gesagt hat: So, die Kamera ist jetzt an. Die war einfach an, ja.“

(037_GD_Polizei).
} 
Videografieren auch immer wieder kritisch als zu starres, die Polizeiarbeit behinderndes, Korsett gesehen werden.

\section{Aufnahmeinhalt}

In Bezug auf die Definitionsmacht von höchster Bedeutung ist der Inhalt der Aufnahme, die durch Wahl des Bildausschnitts (Raumnutzung, Fokus, Zoom, Aufnahmedauer, Kameraführung) und gegebenenfalls die mündliche Kommentierung festgelegt werden und damit eine Inszenierung begründen. Kennzeichnend für diese ist insbesondere, dass meist von außen in die Demonstration hinein gefilmt wird, also aus polizeilicher Perspektive v.a. auf die Teilnehmenden - auf Basis der Erwartung, dass diese auch die potenziellen Straftäter/innen sind. Entgegen der wiederholten Behauptung (als legitimatorisches Argument) ist die Polizei in dieser Perspektive in vielen Demosituationen gerade nicht zentral erfasst. Der dominante Blick ist ein polizeilicher, gerichtet von außen nach innen. Beim Filmen kann eine begleitende Kommentierung (vgl. Tuma 2017, 140 f.) erfolgen, „so eine Art äh Nacherzählung, was wir auf dem Band sehen“ (031_GD). Die Art des Filmens produziert dabei machtvoll Wirkungen: ein im Weitwinkel aufgenommener, geschlossener schwarzer Block wirkt möglicherweise bedrohlicher als Detailaufnahmen von konkreten Personen; eine verwackelte, hektische Kameraführung schafft eine andere Stimmung als eine ruhige. ${ }^{12}$

Zusammenfassend lässt sich zur Durchführung - der Realisierungsstufe - festhalten: Es entstehen Aufnahmen, deren Existenz, Inhalt und Umfang kontingent, aber ausschließlich durch eine polizeiliche Perspektive geprägt sind.

\subsection{Nachbereitung: (Nicht-)Nutzung}

\subsubsection{Das Grundmuster}

Wenn Bilder vorliegen, können diese unmittelbar genutzt werden. In Beweissicherungs- und Dokumentationskraftwagen (BeDoKW) können direkt extrahierte Fahndungsfotos gedruckt und ausgegeben werden. Für das gesamte Material muss aber im Rahmen einer Sichtung eine Verwendung oder Löschung festgelegt werden.

\section{Sichten und Speichern/Löschen}

Eine Vielzahl unterschiedlicher Praxen mit teilweise extrem großen Ermessensspielraum prägen diesen Schritt. Bei Vorliegen von Videodaten steht zuerst die Frage an, ob sie für weitere Zwecke, i.d.R. der Strafverfolgung, genutzt werden. Dazu muss das Material gesichtet werden - vor Ort an der Kamera oder später in der Dienststelle, ggf. auch zentral in einer "Bearbeiterstraße“.

In manchen Einheiten werden Aufnahmen automatisch indexiert, um das selbständige Löschen zu verhindern. Bilder werden dann i.d.R. zentral gespeichert und müssen nach Ablauf einer Frist gelöscht werden, wenn sie nicht als Beweismittel Verwendung finden.

In vielen Fällen liegt die Entscheidung über Speichern/Löschen jedoch bei einzelnen Beamt/innen oder beim Bedo-Trupp. Die Löschung nicht benötigten und ggf. auch nicht erwünschten Bildmaterials (vgl. 5.3.2) kann direkt vor Ort oder nach dem Einsatz bei der Materialsichtung erfolgen; dann hat der

\footnotetext{
${ }^{12}$ Aufschlussreich für eine Analyse der Aufnahmen selbst und ihrer Rezeption wäre sicherlich eine medienwissenschaftlich und kunsthistorisch erweiterte Theorieperspektive, die polizeiliche Videofilme mit andere perspektivischen Inszenierungen von großer Deutungsmacht vergleicht, bspw. mit den bekannten Opferbildern aus der Zeit des Nationalsozialismus, die mehrheitlich von Tätern geschaffen wurden oder mit polizeilichen „Verbrecherbildern“; vgl. dazu Regener $(1999,18)$, die die Fotografiegeschichte (als Vorläufer der Filmgeschichte) auch als „Geschichte zunehmender Symbolisierungen von Differenzen“ begreift.
} 
Filmvorgang keine bürokratischen Prozesse zur Folge. Es kann ein Löschvermerk angelegt werden, allerdings ist dies nicht zwingend:

Da hat man also eine Minute aufgenommen und sagt sich eigentlich: Ach naja, das ist jetzt nichts. Und der eine Kollege löscht das dann und der andere sagt: ... einfach um sich den Vorwurf nicht gefallen lassen zu müssen, so nach dem Motto, naja, was haben sie denn da gelöscht, gibt der eine das halt ab und ein anderer sagt: Du, wenn ich nichts habe, was soll ich da jetzt Archivnummern ausfüllen und Arbeit schaffen? [...] Also beide Wege sind möglich. (022_GD)

In einer anderen Gruppendiskussion mit Mitgliedern der gleichen Bereitschaftspolizeieinheit wird noch deutlicher zu Protokoll gegeben, dass auch eine Komplettlöschung ohne Aktenvermerk erfolgen kann.
„Protokoll wird ganz normal gefertigt, da wird dann einfach dann eingetragen: Bandlöschung wegen irrelevanter Bildaufnahmen. Sollte es dazu kommen, dass nur die Bandansage drauf ist, als einzige Sequenz und eventuell noch mal eine Aufnahme, wo man halt dachte, da passiert gleich was, ja, wird einfach nur gelöscht.“(031_GD)

Wenig überraschend ist daher, dass die Einschätzungen darüber, welcher Anteil der Aufnahmen gelöscht wird, weit auseinandergehen. Mehrere Diskutanten sagen, dass es dabei um die große Mehrheit, bei manchen um fast alle Aufnahmen geht, die sich letztlich als nicht verwertbar erweisen; eine Bedo-Aubilder/in hingegen geht von etwa $10 \%$ nicht verwertbarer Aufnahmen aus.

Maßgeblich für die Entscheidung über Speichern/Löschen und ggf. Weiterbehandlung sind (strafrechtliche) Relevanz, Bearbeitungsaufwand, Speicherplatzbedarf und - mutmaßlich Opportunität (vgl. 5.3.2).

\section{Bearbeitung}

Wenn sich Material als relevant erweist, folgt ein bürokratischer, d.h. nun auch schriftförmiger, aktenbasierter Prozess. Der Vorfall wird dafür mit Bezug auf Rechtsgrundlagen geschildert und interpretiert; weitere Zeugen und Beweismittel, bspw. auch Internetvideos von Demonstrierenden, aufgeführt. In die Akte kommt nicht zwingend das Rohmaterial. Oft erfolgt eine Weiterbearbeitung (Timmermanns 2010, 20), bei der Details „,herausgearbeitet“ werden (035_INT). Dies kann durch die üblichen Videobearbeitungstechniken (Verlangsamen, Zoomen, Aufhellen usw.), durch Hervorhebungen von Einzelaspekten des Geschehens (durch Kreise, Pfeile usw.) und Extraktion von Standbildern geschehen (zur Technologie vgl. Tuma 2017, 146 ff.). Somit erfolgt in der „Form des aktiven Konstruierens von Spuren“ (Tuma 2017, 133) eine zweite Inszenierungsstufe der Vereindeutigung der Evidenz, deren Ziel eine kohärente und gut belegte Erzählung ist, für die „alle Beweismittel so gut wie möglich gesichert wurden und man eben ein sauberes Gerichtsverfahren irgendwann damit durchführen kann“ (031_GD). Das Ziel - durchaus auch als persönliches Motiv einzelner Beamter - ist es, die Verurteilung der Verdächtigen zu erreichen.

Man kann diese Phase als Übergang von einer polizeilichen Beweissicherungslogik zur einer auf Staatsanwaltschaft und Gericht hin orientierten, die Verwendung von Videos als Beweis in Verfahren antizipierenden und inhaltlich auf Herstellung von Eindeutigkeit abzielenden Berichts- und Fixierungslogik verstehen. ${ }^{13}$ Die Akte mitsamt dem Material kann dann der Staatsanwaltschaft zur Strafverfolgung zugeführt werden. Die gelegentlich erfolgende Nachnutzung der Videos als Gedächtnisstütze zur Vorbereitung auf Zeugenaussagen durch filmende Beamt/innen führt dann paradoxerweise zu einer Konvergenz von Sach- und Videobeweis und verstärkt damit noch einmal die Logik von Vereindeutigung und Fixierung in diesem letzten Prozessschritt.

\footnotetext{
${ }^{13}$ Diese Unterscheidung folgt im Grundsatz Jacobsen (2016), die in Ermittlungen die beiden Phasen des polizeilich orientierten und des staatsanwaltlich orientierten Erkenntnisprozesses ausmacht.
} 


\subsubsection{Extreme: Desinformation und Fälschung}

Man kann die aufgeführten Freiheitsgrade und Varianzen als Einfallstor für Willkür und Beliebigkeit deuten und den Begriff der Inszenierung so missverstehen, dass am Ende des Prozesses eine Fälschung steht. Dies wäre tatsächlich irreführend. Der Prozess führt stattdessen über Selektionen, die Alternativen ausschließen und damit Zukunftshorizonte einschränken, dazu, dass nicht Objektivität, sondern Perspektivität entsteht. Gleichwohl ist explizite Desinformation und Fälschung, wie das Verbergen von relevanten Videos oder Szenen, Teil der empirischen Realität. Es gibt keine belastbaren Erkenntnisse zum Ausmaß solcher Praxen, die innerhalb des faktischen Ermessensspielraums den rechtlich legitimierbaren Bereich eindeutig überschreiten und die dem Bereich polizeilicher Devianz zugeordnet werden müssen; verschiedene Hinweise, v.a. einige gut dokumentierte Fälle lassen jedoch auf die Existenz eines veritablen Dunkelfeldes schließen.

So gab es im Jahr 2013 in Leipzig eine kleine, friedliche linksradikale Demonstration, die augenscheinlich abgefilmt wurde. Sowohl mehrfach gegenüber der Anmelderin als auch in einem von ihr angestrengten Feststellungsverfahren erklärte die Polizei, nicht gefilmt zu haben. Später jedoch führte sie ein Verfahren gegen die Anmelderin auf Basis von polizeilichem Filmmaterial dieser Demonstration, dessen Anfertigung anschließend gerichtlich für nicht rechtmäßig erklärt wurde (VG Leipzig Az.: $1 \mathrm{~K}$ 222/13). Dieses Geschehen verdeutlich den verbreiteten polizeilichen Unwillen zu versammlungsfreundlichem und kooperativem Agieren (zumindest mit dem politischen Spektrum der hier Demonstrierenden), die Praxis illegalen Filmens und zugleich die offensive Irreführung Betroffener über eingriffsintensive Maßnahmen.

Noch schwerwiegender sind Fälle aus Dresden im Kontext von Protesten gegen Rechtsextreme (2011). ${ }^{14}$ Der Jenaer Jugendpfarrer König war angeklagt worden, im Protest aufwieglerisch agiert und zu Gewalt aufgerufen zu haben. Erst im Laufe des Prozesses wurden der Verteidigung durch einen Zufall ihr bisher von der Polizei vorenthaltene Schriftstücke und ungeschnittene Videos bekannt, die den wohl abgesprochenen Aussagen der Polizeizeugen und den zuvor präsentierten suggestiv geschnittenen Videos und fehlerhaften Transkriptionen grundsätzlich widersprachen. Nach den Funden wurde der Prozess zunächst ausgesetzt und schließlich eingestellt. Gegen den verantwortlichen Polizisten Maik U. wurde wegen des Vorwurfs der Manipulation des Videomaterials ermittelt. Gleiches wurde ihm im Fall „Tim H.“ vorgeworfen. Die Anklage wegen Landfriedensbruch versuchte nachweisen, dass nur eine Person mit Megaphon anwesend war - der Angeklagte. Das später hinzugezogene Rohmaterial (auch dem Fund der Anwälte Königs zu verdanken), zeigte fünf (!) Personen mit Megaphon. Während entscheidende Szenen fehlten, befand sich in der Akte eher skurriles Material, bspw. Zusammenschnitte von Gewaltszenen des Tages sowie mit Musik unterlegte und mit gelb-roter Comic-Schrift kommentierte Clips vor einem arrangierten Hintergrundbild einer brennenden Barrikade. Die Anklage musste fallen gelassen werden, der Richter bezeichnet das bearbeitete Polizeivideo als "praktisch nicht brauchbar“.

In beiden Fällen konnte die fingierte Anklage nur durch den zufälligen Aktenfund widerlegt werden und weil die Angeklagten Unterstützung mobilisieren konnten. Tim H. beschäftigte unter erheblichem Kostenaufwand eine Person zur Katalogisierung der relevanten Filmszenen aus zweihundert Stunden Material und holte so die unterlassene Ermittlungsarbeit der Polizei nach. Personen ohne diese mobilisierbare Beschwerdemacht wären wohl verurteilt worden.

Die Fälle verdeutlichen, wie Strafbedürfnisse und der Überführungswunsch beim Übergang von der Beweissicherungslogik zur Fixierungslogik zum Verschwinden von Deutungsunklarheiten führen. In beiden Fällen ist für die Eindeutigkeit der polizeilichen Argumentation nicht dienliches Material dem

\footnotetext{
${ }^{14}$ Die Rekonstruktion beider Fälle folgt umfangreichen Presseberichten, Urteilen, Gesprächen mit Prozessbeteiligten, Videomaterial sowie Dokumenten in Eisenberg et al. (2014).
} 
Gericht vorenthalten worden. Der noch weiter gehende Schritt, dass zum Latenzschutz und zur Vermeidung von in-the-job-trouble (Waddington 1994) Material als „unverwertbar“ gelöscht wird, welches deviantes Polizeiverhalten erfasst, ist entsprechend höchst plausibel.

\section{Risikokonstellationen für over-watching}

Das vorliegende Material ist wegen der Dominanz von Selbstzeugnissen nicht geeignet, eine umfassende Erklärung zu liefern, wann genau welche der Optionen in der Einsatzpraxis gewählt werden. Doch es liefert, flankiert durch Sekundärliteratur Hinweise auf Faktoren, die ,over'- oder ,underpolicing' wahrscheinlicher machen, bzw. zur Risikokonstellation für ,over'- oder ,underwatching' beitragen.

Von Relevanz ist erstens die Einordnung des ,polizeilichen Gegenübers' in Normalitätsklassen (Ullrich 2017). Die Daten verdeutlichen Auswirkungen v.a. in der Planungsphase in der Deutung antagonistischer Bewegungen als weniger legitim (vgl. Winter 1998b). Dies hat zur Folge, dass das Policing der Nicht-„,Normaldemonstranten“ einem größeren Aufmerksamkeitspotenzial unterliegt, Kameras eher bereitgehalten und leichter einsetzbar werden. Dies verdeutlichen die genannten Gefahrensignale, die einen, politischen' Ermessensspielraum offenbaren (Ullrich 2017; Arzt und Ullrich 2016). Wie schon in klassischen Studien zum soziologischen Ermessen zeigt sich, dass die ,gute Gesellschaft', , das bürgerliche Klientel' weniger Sanktionierung erfährt als das „police property“ (Lee 1981), zu dem u.a. radikale politische Gruppen gehören (Waddington 2007, S. 22):

Und das bringt die Beamten [...] auch in einen Zwiespalt, ne? Dort sitzt ein bürgerliches Klientel auf der Straße, wo ich sage: Die begehen alle eine Straftat. Theoretisch als Polizei muss ich jetzt handeln [...]. Politisch gesehen ist es sicher gerade angesehen, dass man die dort sitzen lässt [...]. Dann geht man von dem polizeilichen Notstand aus, dann gibt es da sicher Wege, dass man das dann nicht macht. (056 GD)

Polizei wird hier zum Legitimitätsakteur. Diesen Befund stützen auch die durchgeführten Demonstrationsbeobachtungen. Zwei Merkmale sind danach von herausragender Bedeutung für die Anwesenheit/Nutzung von Videoüberwachungstechnik: größere Versammlungen und solche von antagonistischen Protestgruppen (meist radikale Linke, Rechtsextreme sowie Konfrontationslagen) werden häufiger potenziell oder real videografiert (Knopp und Müller-Späth 2017).

Zum zweiten wurde verschiedentlich zu Protokoll gegeben, dass Kommunikationsoffenheit der Demonstrierenden gegenüber der Polizei mit Nachsicht belohnt wird (vgl. a. della Porta, Fillieule, und Reiter 1998):

Und ein wesentlicher Beitrag dafür, dass die Einschreitschwelle hoch ist, ist zum Beispiel die Kooperationsbereitschaft des Veranstalters. Wenn der im Vorfeld schon kooperationsbereit ist, heißt es für die Polizei im Einsatz, dass man ein paar Sachen hinnimmt, auch mal drüber wegguckt, sich mal, ich hätte fast gesagt, die Schnürsenkel zumacht bei Kleinigkeiten [...]. Andersrum natürlich, wenn die Kooperationsbereitschaft überhaupt nicht gegeben ist, der hat seine Anmeldungen abgegeben, und alles andere ist ihm egal, dann ist die Polizei auch mal geneigt [...] eher einzugreifen. (037_GD)

In der Kooperationsbereitschaft drückt sich die Anerkennung der polizeilichen Autorität aus, und diese wird belohnt. Auch in kodifizierten Gefährderklassifikationen gehört Kooperationszurückhaltung zu den Merkmalen für die rote Kategorie „Störer“.

Ein dritter Faktor wird in den Situationen deutlich, in denen die Aufnahmeentscheidung nicht intuitionsbasiert, sondern aufgrund bestimmter Umstände bewusst reflexiv erfolgt. Dazu zwei Beispiele aus einer Reihe von Sequenzen in mehreren Gruppendiskussionen über Einschaltsituationen bei überwachungskritischen Demonstrationen: 
„es ist immer [...] stark abhängig davon, wie der Demonstrationsanlass ist und-, ja gerade bei diesen datenschutzrechtlichen Demonstrationen sollte man halt besonders darauf achten, wann man filmt [...]. Also dementsprechend ist der Zeitpunkt, an dem ich anfange zu filmen, [...] der liegt wesentlich weiter hinten als bei anderen Demonstrationen. [...] Da achtet man dann als Polizeibeamter dann auch schon sehr, sehr stark darauf. Darf ich das jetzt wirklich oder nicht? ... "(031_GD)

Eine Deutung für die angesprochene Zurückhaltung beim Filmen von Datenschützer/innen drängt sich auf: Sensibilität für Personen, die gegen Überwachung selbst besonders sensibel sind. Doch die Frage am Ende der Sequenz verdeutlicht, dass hier eigentlich Rechtsunsicherheit herrscht und die Rechtmäßigkeit des eigenen Routinehandelns als prekär begriffen wird, insbesondere wo das ,polizeiliche Gegenüber' in diesem Fall auch über Wissen sowie besondere Beschwerdemotive und damit Beschwerdemacht verfügt. Noch besser verdeutlicht diese Mehrdeutigkeit polizeilicher Zurückhaltung die zweite Sequenz (aus einer Gruppendiskussion in einem anderen Bundesland):

„Ja, also prinzipiell ist es schon die [...] Entscheidung des Beamten, [...] aber es gibt natürlich auch [...] Einsätze, wo von vornherein schon ein äußerstes Deeskalationsprinzip gefahren wird, wo man sagt, wir wollen uns hier absolut gar nichts vorwerfen lassen, wo dann auch schon gesagt wird, na ja, die Kameras sollten nach Möglichkeit ausbleiben, aber das ist äußerst selten, und so lange das der BedoBeamte verantworten kann, lässt er die dann auch aus" (034_GD)

Ein hochrangiger Beamter nennt auch explizit politische Erwägungen als Grund für Zurückhaltung beim Filmen trotz Vorliegen der Eingriffsvoraussetzungen. Dies macht den Umkehrschluss plausibel, dass im Normalfall, der nicht besondere Reflexionen motiviert, genau diese Abwägung nicht (oder mit weitaus geringerer Bedeutung) getroffen wird.

Diese Problematik wird auf Praxisebene womöglich noch dadurch verschärft, dass Fußball das zweite große Einsatzgebiet der Bereitschaftspolizei ist. Trotz deutlich höherer Eingriffsschwellen im Versammlungsrecht, werden in den Diskussionen beide Einsatzgebiete immer wieder auch als handlungspraktisch identische beschrieben. Eine Diffusion von weniger reglementierten Praxen im Fußball in das stärker verregelte Feld der Versammlungen muss angenommen werden.

\section{Schlussfolgerungen}

Videoüberwachung von Demonstrationen muss als nicht deterministischer Prozess verstanden werden. Er besteht aus einer Abfolge polizeilicher Entscheidungen mit jeweiligen Anschlussoptionen, die von der Potenzialbestimmung in der Vorbereitungsphase über die polizeilich orientierte Durchführungsphase bis zur staatsanwaltschaftlich orientierten, auf Verurteilung abzielenden Fixierung in der Nachbereitungsphase reicht.

Die Legitimierung des Prozesses - in den Transkripten als inhaltlich vom Modus des Praxisberichts abweichende Legitimationserzählung präsent - kreist um die Begriffe Rechtssteuerung, Neutralität und Objektivität. Videoüberwachung ist aus polizeilicher Sicht von rechtschaffenen Bürger/innen nicht zu fürchten, denn sie finde sparsam und streng nach den Vorschriften Anwendung (Rechtsdeterminiertheit) und biete eine unparteiische, alle Seiten erfassende (Neutralität) und verlässliche Darstellung relevanter Vorkommnisse (Objektivität). ${ }^{15}$ Die empirische Analyse der Konstruktion von Evidenz im Videoüberwachungsprozess offenbart ein gegenteiliges Bild: Existenz, Inhalt und Bearbeitung der Videos sind von polizeilichen Entscheidungen und polizeilicher Perspektivität geprägt. Angesichts dieser verketteten Struktur der Produktion von Deutungsmacht wurde dafür der Begriff der polizeilichen Definitionsmachtkette vorgeschlagen.

\footnotetext{
${ }^{15}$ Tuma (2017, S. 158 ff.) kommt zum abweichenden Befund hohen Kontingenzbewusstseins. Das liegt wahrscheinlich an Tumas Hauptquelle, einem spezialisierten forensischen Videoanalysten - im Gegensatz zu den hier dominierenden polizeilichen „Handarbeitern“ (Behr 2000a).
} 
Die polizeiliche Definitionsmacht liegt in der Vielzahl möglicher Selektionen für einzelne Beamt/innen und die Polizeiorganisation, mithin im soziologischen Ermessensspielraum. Nur zu einem geringen Teil sind die jeweiligen Entscheidungen rechtsdeterminiert, auch wenn gesetzliche Definition, organisationales Selbstbild und Ausbildung in der Polizei diese Vorstellung hegen und pflegen (Winter 1997). Die Rechtsdeterminiertheit erweist sich jedoch angesichts der dargestellten Freiheitsgrade ebenso wie die postulierte Objektivität empirisch als Fiktion, wenngleich als aufgrund der gesetzlichen Definition quasi notwendige. Das Recht ist zwar als ein Motiv polizeilichen Handelns unschwer zu erkennen. Aber es ist nur ein Motiv unter vielen. Das Recht erweist sich vielmehr als doppelte Rechtfertigungsstruktur: zum einen für Handeln in Einsatzsituationen und zum anderen als Argumentationsreservoir für ein Idealbild polizeilicher Videoüberwachung, eine von Widersprüchen, Ambivalenzen und Devianz bereinigte Schauseitenversion der tatsächlichen Praxen.

Den praktischen Prozess kennzeichnet insbesondere, dass die bei jedem Schritt bestehende Kontingenz im weiteren Verlauf tendenziell unsichtbar wird. Wenn eine Aufnahmesituation vorliegt, ist nicht mehr von Belang, warum Kameras anwesend waren. Wenn fixierte Evidenz vorliegt, bedarf es mindestens aktiven Zweifelns und des Suchens nach Gegenevidenz, um die scheinbar objektiven Bilder zu hinterfragen. Dies funktioniert und erscheint plausibel, weil der Prozess weitgehend ein auf Sachtechnik (Kameras, Computer, Software u.v.m.) als in routinierte Praxisvollzüge eingebetteter Ressource gestützter ist (Schulz-Schaeffer 1999). Schritte im Prozess bringen Objektivationen hervor, die im nächsten Schritt als selbstverständlich vorausgesetzt werden, weil sie in ihrer

Technikvermitteltheit als nicht durch Subjektivität Getrübtes erscheinen, sondern vielmehr von einer „Aura der Authentizität“ (Bredekamp 2004, S. 45) umgeben sind. Der Gesamtprozess ist also ein Fall von Blackboxing, bei dem der Output den Vorgang als solchen rechtfertigt und dessen

Zustandekommen der Hinterfragung enthebt (Latour 1999, S. 183 f., 304). Damit ist der Prozess der Definitionsmachtkette zugleich eine durch die Distanziertheit des skopischen Mediums mögliche „Abstraktion vom Sozialen“ (Belina und Wehrheim 2011). Der letztlich in die Strafakte eigehende bearbeitete Videofilm abstrahiert weitgehend von seinen Ermöglichungsbedingungen auf den isolierten Rechtsbruch hin. Der soziale Konflikt hinter dem Protestereignis, die geteilte Konfliktgeschichte von Polizei und Demonstrierendengruppen mit ihren Feindbildern und auch die Gesamtsituation auf der Demonstration sind vom Standpunkt des Strafrechts nicht mehr relevant ein dokumentierter Rechtsbruch ist zu verfolgen. Doch die Sanktionierungswahrscheinlichkeit ist zuungunsten antagonistischer und weniger beschwerdemächtiger Gruppen ungleich verteilt.

Die Videoerfassung ist eine Kriminalisierungsvoraussetzung, die im Extrem in ungerechtfertigter Verfolgung resultieren kann, wie die von Überführungslogik und Strafwünschen geprägten Manipulationen dokumentieren.

Die eigentliche Bedeutung des Dargestellten erschließt sich jedoch erst im sozialen Kontext der Videoüberwachung. Im institutionellen Zusammenhang werden die Folgen möglichen Kumulierens von Perspektivität und Partialität deutlich. Polizeiliche Autoritätssicherungsstrategien, Strafwünsche und Feindbilder gegen bestimmte Gruppen werden zu selbsterfüllenden Prophezeiungen. Die Nähe zwischen Polizei und Staatsanwaltschaft verstärkt die Bedeutung der Deutungen erster. Durch Sichtbarkeitsasymmetrien ${ }^{16}$ und ungleiche Durchsetzungschancen im Protestgeschehen selbst und Unterschiede im Standing in den Medien, dem wohl zentralen Adressaten von Protest, muss eine sehr hohe Schwelle überschritten werden, um die Rolle der Polizei als primary definer fallbezogen in Frage zu stellen. Entsprechend wäre von einer doppelten Definitionsmacht zu sprechen: hinsichtlich der strafrechtlichen Kriminalisierungs- und der medialen Stigmatisierungsmacht. Aspekte dieses

\footnotetext{
${ }^{16}$ Vermummungs- und Uniformierungsverbot für Demonstrierende vs. polizeiliche Uniformierung mit Tendenz der Unkenntlichkeit im gegenwärtigen riot gear, fehlende Kennzeichnung und - auch in den Projektdaten vorherrschend - strikte Ablehnung einer individuellen Kennzeichnungsflicht.
} 
Prozesses detaillierter zu untersuchen, bspw. wie der Videobeweis den Verlauf von Strafverfahren ändert, bleibt künftiger Forschung vorbehalten, ebenso die rechtssoziologische Frage, inwiefern das Recht einen Beitrag zur Verhinderung oder Minimierung selektiver Sanktionierung leisten kann.

Wenn man also Videoüberwachung als Machtverstärker (Ullrich 2011, S. 38) hegemonialer Ordnungsvorstellungen begreift, stellt sich die Anschlussfrage nach möglichen Änderungsrichtungen derselben. Hier ist zu wiederholen, dass die polizeiliche Definitionsmacht nicht unangefochten ist. Betroffene von Videoüberwachung setzen sich mit Neutralisierungstechniken zur Wehr, bis hin zur Produktion komplexer Gegenevidenz (Tuma 2017, S. 160 ff.); gelegentlich können mediale Skandalisierungen die Definitionsmachtkette durchbrechen (Greer und McLaughlin 2010). Angesichts gegenwärtiger Tendenzen der Normalisierung des Ausnahmezustands (u.a. Dießelmann 2015) ist eher eine Entgrenzungslogik zu erwarten. Womöglich ist in dieser nicht nur die Rechtsbindung der Polizei als solche fragil, sondern bröckelt sogar die Fiktion derselben und damit deren begrenztes normatives Potenzial.

\section{Literatur}

Arzt, Clemens, und Peter Ullrich. 2016. „Versammlungsfreiheit versus polizeiliche Kontroll- und Überwachungspraxis“. vorgänge. Zeitschrift für Bürgerrechte und Gesellschaftspolitik 55 (1): 46-60.

Backes, Otto, Rainer Dollase, und Wilhelm Heitmeyer. 1998. „,Wie groß ist die Fremdenfeindlichkeit in der Polizei?' Eine Analyse zu Risikokonstellationen im Polizeialltag“. Herausgegeben von Institut für interdisziplinäre Konflikt- und Gewaltforschung. IKG Newsletter 9 (1): 4-11.

Becker, Howard S. 1967. „Whose Side Are We On?" Social Problems 14 (3): 239-47.

Behr, Rafael. 2000a. Cop culture - der Alltag des Gewaltmonopols. Männlichkeit, Handlungsmuster und Kultur in der Polizei. Wiesbaden: Leske + Budrich.

- - . 2000b. „Funktion und Funktionalisierung von Schwarzen Schafen in der Polizei. Arbeitsskizze aus der empirischen Polizeiforschung". Kriminologisches Journal 32 (3): 219-29.

-- - 2006. Polizeikultur. Routinen - Rituale - Reflexionen. Bausteine zu einer Theorie der Praxis der Polizei. Wiesbaden: VS Verlag für Sozialwissenschaften.

Belina, Bernd, und Jan Wehrheim. 2011. „,Gefahrengebiete'. Durch die Abstraktion vom Sozialen zur Reproduktion gesellschaftlicher Strukturen“. Soziale Probleme 23 (1): 207-30.

Bredekamp, Horst. 2004. „Bildakte als Zeugnis und Urteil“. In Mythen der Nationen. 1945 - Arena der Erinnerung, herausgegeben von Monika Flacke, 29-68. Mainz: Verlag Philipp von Zabern.

Brusten, Manfred. 1971. „Determinanten selektiver Sanktionierung durch die Polizei“. In Die Polizei. Soziologische Studien und Forschungsberichte, herausgegeben von Johannes Feest und Rüdiger Lautmann, 31-70. Opladen: Westdeutscher Verlag.

Dießelmann, Anna-Lena. 2015. Ausnahmezustand im Sicherheits- und Krisendiskurs. Eine diskurstheoretische Studie mit Fallanalysen. Siegen: Universitätsverlag Siegen.

Earl, Jennifer, Sarah A. Soule, und John D. McCarthy. 2003. „Protest under Fire? Explaining the Policing of Protest“. American Sociological Review 68 (4): 581-606.

Eggert, Nina, Ruud Wouters, Pauline Ketelaars, und Stefaan Walgrave. 2016. „Preparing for Action. Police Deployment Decisions for Demonstrations". Policing and Society, 1-12.

Eisenberg, Johannes, Lea Voigt, und Manuel Vogel, Hrsg. 2014. Der Prozess gegen den Pfarrer Lothar König - Antifaschismus als Feindbild. Hamburg: LAIKA.

Feest, Johannes, und Erhard Blankenburg. 1972. Die Definitionsmacht der Polizei. Strategien der Strafverfolgung und soziale Selektion. Studienbücher zur Sozialwissenschaft 1. Düsseldorf: Bertelsmann Universitätsverlag.

Fekjær, Silje Bringsrud, Otto Petersson, und Gunnar Thomassen. 2014. „From Legalist to Dirty Harry. Police Recruits' Attitudes towards Non-Legalistic Police Practice“. European Journal of Criminology 11 (6): 745-59. 
Gillham, Patrick F., und John A. Noakes. 2007. „,,More than a March in a Circle'. Transgressive Protests and the Limits of Negotiated Management". Mobilization: An International Journal 12: 341-57.

Greer, Chris, und Eugene McLaughlin. 2010. „We Predict a Riot? Public Order Policing, New Media Environments and the Rise of the Citizen Journalist". British Journal of Criminology 50 (6): 1041-1059.

Haggerty, Kevin D., und Richard V. Ericson. 2000. „The Surveillant Assemblage“. British Journal of Sociology 51 (4): 605-622.

Hunold, Daniela. 2015. Polizei im Revier. Polizeiliche Handlungspraxis gegenüber Jugendlichen in der multiethnischen Stadt. Berlin: Duncker \& Humblot.

Jacobsen, Astrid. 2016. „Bericht über die Forschung zur ,Logik polizeilicher Ermittlung““. Forschungsbericht. Polizeiakademie Niedersachsen.

Jasch, Michael. 2002. Perspektiven der polizeilichen Entscheidungsmacht. Strafverfahrensabschluss und Polizei in Deutschland und England. Norderstedt: Books on Demand.

Kammerer, Dietmar. 2008. Bilder der Überwachung. Frankfurt am Main: Suhrkamp.

Knopp, Philipp, und Frederike Müller-Späth. 2017. „Protestereignisse und Videoüberwachung in Berlin. Eine ethnografische Studie“. IPB Working Papers. Institut für Protest- und Bewegungsforschung. https://protestinstitut.eu/ipb-working-papers/.

Knopp, Philipp, und Peter Ullrich. 2016. „Kampf um die Bilder. Videoüberwachung und Gegenüberwachung von Demonstrationen in Österreich“. Juridikum. Zeitschrift für Kritik, Recht, Gesellschaft, Nr. 4: 527-37.

Knorr-Cetina, Karin. 2012. „Skopische Medien. Am Beispiel der Architektur von Finanzmärkten“. In Mediatisierte Welten. Beschreibungsansätze und Forschungsfelder, herausgegeben von Friedrich Krotz und Andreas Hepp, 167-95. Wiesbaden: VS Verlag für Sozialwissenschaften.

Koranyi, Johannes, und Tobias Singelnstein. 2011. „Rechtliche Grenzen für polizeiliche Bildaufnahmen von Versammlungen“. Neue Juristische Wochenschrift, Nr. 3: 124-28.

Kühl, Stefan. 2014. Ganz normale Organisationen. Zur Soziologie des Holocaust. Berlin: Suhrkamp Verlag.

Kutscha, Martin. 2011. „Demonstrationen auf dem Bildschirm der Polizei“. Kritische Justiz, Nr. 2: 22332.

Latour, Bruno. 1999. Pandora's Hope. Essays on the Reality of Science Studies. Cambridge, Massachusetts: Harvard University Press.

Lee, J A. 1981. "Some Structural Aspects of Police Deviance in Relations with Minority Groups“. In Organizational Police Deviance. Its Structure and Control, herausgegeben von Clifford D. Shearing, 49-82. Butterworths.

Loftus, Bethan. 2010. „Police occupational culture. Classic themes, altered times“. Policing and Society: An International Journal of Research and Policy 20 (1): 1-20.

Luhmann, Niklas. 1976. Funktionen und Folgen formaler Organisation. Berlin: Duncker \& Humblot. McPhail, Clark, David Schweingruber, und John McCarthy. 1998. „Policing Protest in the United States: 1960-1995“. In Policing Protest. The Control of Mass Demonstrations in Western Democracies, herausgegeben von Donatella della Porta und Herbert Reiter, 49-69. Minneapolis: University of Minnesota Press.

Porta, Donatella della, Olivier Fillieule, und Herbert Reiter. 1998. „Policing Protest in France and Italy: From Intimidation to Cooperation?" In The Social Movement Society. Contentious Politics for a New Century, herausgegeben von David Meyer und Sidney Tarrow, 111-30. Lanham: Rowman \& Littlefield Publishers.

Regener, Susanne. 1999. Fotografische Erfassung. Zur Geschichte medialer Konstruktionen des Kriminellen. München: Wilhelm Fink Verlag.

Schmitt-Beck, Rüdiger. 1990. „Über die Bedeutung der Massenmedien für soziale Bewegungen“. Kölner Zeitschrift für Soziologie und Sozialpsychologie 42 (4): 642-62.

Schulz-Schaeffer, Ingo. 1999. „Technik und Dualität von Ressourcen und Routinen“. Zeitschrift für Soziologie 28 (6): 409-28. 
Singelnstein, Tobias. 2014. „Körperverletzung im Amt durch Polizisten und die Erledigungspraxis der Staatsanwaltschaften - aus empirischer und strafprozessualer Sicht". Neue Kriminalpolitik 25 (1): 15-27.

Skolnick, Jerome H. 1966. Justice Without Trial. Law Enforcement in Democratic Society. New Orleans: Quid Pro, LLC.

Strauss, Anselm L., und Juliet M Corbin. 1998. Basics of Qualitative Research. Techniques and Procedures for Developing Grounded Theory. Thousand Oaks: Sage.

Timmermanns, Daniela. 2010. „Der neue Beweissicherungs- und Dokumentationskraftwagen (BeDoKw)“. Polizeispiegel 44 (12): 19-20.

Tuma, René. 2017. Videoprofis im Alltag. Die kommunikative Vielfalt der Videoanalyse. Wiesbaden: Springer Fachmedien.

Ullrich, Peter. 2011. Gesundheitsdiskurse und Sozialkritik - Videoüberwachung von Demonstrationen. Zwei Studien zur gegenwärtigen Regierung von sozialen Bewegungen und Protest. Wissenschaftliche Texte des DJI. München: Deutsches Jugendinstitut. http://www.dji.de/fileadmin/user_upload/bibs/9_14638_Videoueberwachung_Ullrich_2012. pdf.

- - . 2014. „Protest und technische Überwachung. Das Beispiel Videoüberwachung“. Forschungsjournal Soziale Bewegungen 27 (4): 40-50.

- - . 2017. „,Normalbürger' versus ,Krawalltouristen'. Polizeiliche Kategorisierungen von Demonstrationen zwischen Recht und Soziologischem Ermessen“. In Empirische Polizeiforschung XX. Polizei und Minderheiten, herausgegeben von Karlhans Liebl, 61-97. Frankfurt am Main: Verlag für Polizeiwissenschaft.

- - . 2018 (im Druck). „Polizei im/unter Protest erforschen. Polizeiforschung als Entdeckungsreise mit Hindernissen". In Polizei und Gesellschaft. Transdisziplinäre Perspektiven zu Methoden, Theorie und Empirie reflexiver Polizeiforschung, herausgegeben von Christiane Howe und Lars Ostermeier. Wiesbaden: VS Verlag für Sozialwissenschaften.

Ullrich, Peter, und Gina Rosa Wollinger. 2011. „A Surveillance Studies Perspective on Protest Policing. The Case of Video Surveillance of Demonstrations in Germany". Interface. A journal for and about social movements 3 (1): 12-38.

Waddington, David P. 2007. Policing Public Disorder. Theory and Practice. Cullompton: Willan. Waddington, P. A. J. 1994. Liberty and Order. Public Order Policing in a Capital City. London: UCL Press.

Willems, Helmut, Roland Eckert, Harald Goldbach, und Toni Loosen. 1988. Demonstranten und Polizisten. Motive, Erfahrungen und Eskalationsbedingungen. DJI Forschungsbericht. München: Verlag Deutsches Jugendinstitut e.V./Juventa.

Wilson, Dean Jonathon, und Tanya Serisier. 2010. „Video Activism and the Ambiguities of CounterSurveillance". Surveillance \& Society 8 (2): 166-80.

Wilson, James Q. 1968. Varieties of Police Behavior. The Management of Law and Order in Eight Communities. Cambridge, Mass.: Harvard University Press.

Winter, Martin. 1997. Die Polizei - autonomer Akteur oder Herrschaftsinstrument? (Die Halleschen Graureiher 97-3). Bd. 1997-3. Der Hallesche Graureiher. Halle: Institut für Soziologie, Universität Halle.

- - . 1998a. „Police Philosophy and Protest Policing in the Federal Republic of Germany, 19601990". In Policing Protest. The Control of Mass Demonstrations in Western Democracies, herausgegeben von Donatella della Porta und Herbert Reiter, 188-212. Minneapolis: University of Minnesota Press.

- - . 1998b. Protest Policing und das Problem der Gewalt. Die Halleschen Graureiher 98-5. Halle: Institut für Soziologie, Universität Halle.

Wisler, Dominique, und Marco Giugni. 1999. „Under the spotlight. The impact of media attention on protest policing". Mobilization. An International Journal 4 (2): 171-87.

Wood, Lesley J. 2014. Crisis and Control. The Militarization of Protest Policing. London/Toronto/New York: Pluto Press, Between the Lines. 
Peter Ullrich Dr. phil. Dr. rer med., ist Ko-Leiter des Forschungsbereichs „Soziale Bewegungen, Technik, Konflikte“ am Zentrum Technik und Gesellschaft der TU Berlin. Er ist Sprecher des Arbeitskreises „Soziale Bewegungen und Polizei“. Kontakt: https://doi.org/http://textrecycling.wordpress.com, ullrich@ztg.tu-berlin.de 